2 Yang $Y$, Bai-Lin W, Shen Y. A pilot study on the diagnostic performance of DSM-IV and DSM-V for autism spectrum disorder. N A J Med Sci 2011; 4 $116-23$.

Michael Fitzgerald, Professor of Child Psychiatry, Trinity College Dublin, Ireland. Email: fitzi@iol.ie

doi: $10.1192 / \mathrm{bjp} .201 .1 .74 \mathrm{~b}$

\section{The Department of Health and the Equality} Act 2010

In their otherwise excellent review of the Equality Act 2010 and mental health, ${ }^{1}$ the authors did not highlight how the Department of Health currently discriminates against people with mental health problems.

The National Health Service (NHS) constitution has incorporated the Equality Act in terms of access to NHS care, including on the grounds of disability. However, a fundamental right of the constitution is that of choice. Section 2a states 'You have the right to make choices about your NHS care and to information to support these choices. The options available to you will develop over time and depend on your individual needs. ${ }^{2}$

Since April 2009, patients have had a right to choose the service that provides their treatment when they are referred for their first out-patient appointment with a consultant-led team. Patients can review outcome data, specialist expertise and user feedback for a service, discuss it with their general practitioner, and be referred for an elective medical or surgical problem to any NHS consultant-led service across the country. However, the Department of Health excludes patients detained under the Mental Health Act 1983, military personnel and prisoners. It also excludes services where speed of access to diagnosis and treatment is important, for example emergency admissions and maternity services. However, under this clause it also excludes elective mental health services. This appears to be discriminatory under the Equality Act for people with mental health problems who are disabled by their disorder. So anyone with a mental disorder who is disabled and has had treatment locally cannot by right obtain a referral to a specialist mental health service. Most National Institute for Health and Clinical Excellence guidelines on mental disorders envisage stepped care. Where treatment has failed, the next step is onward referral to more intensively delivered cognitive-behavioural therapy (e.g. more frequent, longer sessions with more experienced therapists) or to specialised pharmacological advice that may not be available from a local community mental health team or psychology service.

Patients with mental disorders who are disabled therefore have the right to choose where they have treatment for their cancer, for example, but not for their mental disorder. Access depends entirely on the vagaries of local funding panels. The legal right to choice of elective care should be extended to mental health services, or withdrawn from surgery and medicine. The present discrimination is unconscionable.

1 Lockwood G, Henderson C, Thornicroft G. The Equality Act 2010 and mental health. Br J Psychiatry 2012; 200: 182-3.

2 Department of Health. The Handbook to the NHS Constitution. Department of Health, 2012.

David Veale, FRCPsych, Consultant Psychiatrist, South London and Maudsley NHS Foundation Trust, and Institute of Psychiatry, King's College London, UK. Email: David.Veale@kcl.ac.uk

doi: 10.1192/bjp.201.1.75

\section{Monitoring cardiometabolic risk in schizophrenia}

I commend De Hert et $a l^{1}$ for their attempt to clarify appropriate monitoring for cardiometabolic risk in schizophrenia. I agree that cardiometabolic risk is one important consideration for these patients.

I note that their findings included generally low scores for the rigour of existing guidelines and a lack of evidence of long-term patient outcomes. It is perhaps a little surprising then that they nevertheless make recommendations on what appears to be less than robust evidence.

I have previously expressed concerns that cardiometabolic screening programmes of this type are unevaluated and that the benefits are unknown, as are the risks, which seem to have received little attention. ${ }^{2}$

The authors quite rightly highlight that guidelines can be biased because of lack of scientific evidence, but the evidence they present to support their protocol appears to fall well short of the levels of evidence recommended for interventions. ${ }^{3}$ I can find no evidence that patients will benefit from such a protocol, and none that they will not be harmed.

I also note that their suggested protocol differs from National Institute for Health and Clinical Excellence (NICE) quality and outcomes framework standards for mental illness (www.nice. org.uk/aboutnice/qof/indicators.jsp) and NICE guidelines for lipid modification, both of which recommend primary preventive screening for patients aged over $40 .^{4}$

I wish to support the notion that interventions should be evaluated before implementation. ${ }^{3}$

1 De Hert M, Vancampfort D, Correll CU, Mercken V, Peuskens J, Sweers K, et al. Guidelines for screening and monitoring of cardiometabolic risk in schizophrenia: systematic evaluation. Br J Psychiatry 2011; 199: 99-105.

2 Reed PF. Let's target screening more effectively. Psychiatrist 2010; 34 $540-1$.

3 Oxford Centre for Evidence-Based Medicine. CEBM (Centre for EvidenceBased Medicine) Levels of Evidence. CEBM, 2012 (http://www.cebm.net/ index.aspx?0=5653)

4 National Institute for Health and Clinical Excellence. Lipid Modification: Cardiovascular Risk Assessment and the Modification of Blood Lipids for the Primary and Secondary Prevention of Cardiovascular Disease (Clinical Guideline 67). NICE, 2008.

Paul F. Reed, Consultant Psychiatrist, Lancashire Care NHS Foundation Trust, UK. Email: paul.reed@lancashirecare.nhs.uk

doi: 10.1192/bjp.201.1.75a

Physical care of people with severe mental illness is an important clinical issue, as the potential health benefits of cardiovascular disease prevention for the general population are astonishing. Each year, cardiovascular disease kills about 20 million people, including 10 million prematurely (before the age of 65 years) and inflicts high morbidity, disability and socioeconomic costs. ${ }^{1}$ This problem is more pronounced in schizophrenia, with standardised mortality rates (SMRs) of 2.7 for diabetes and 2.3 for cardiovascular disease. ${ }^{2}$

Cardiovascular mortality increased in schizophrenia from 1976 to 1995 , with the greatest increase in SMR in men from 1991 to $1996 .{ }^{3}$

In the current climate of austerity in the National Health Service and internationally, it is interesting to know that in high-income countries, preventing or postponing 100 cases has been reported as saving about US\$1 million ( $£ 0.6$ million, $€ 0.7$ million). ${ }^{4}$ 